\title{
INTERNAL CAROTID ARTERY BLOOD BLISTER-LIKE ANEURYSM
}

\author{
Joacil Carlos da Silva ${ }^{1}$, Igor Vilela Faquini ${ }^{2}$, Matheus Augusto Pinto Kitamura ${ }^{2}$, \\ Hildo Rocha Cirne de Azevedo-Filho'
}

Aneurysms located at nonbranching sites in the supraclinoid internal carotid artery (ICA) were originally characterized, in 1986, as aneurysms protruding from the dorsal wall of the ICA', but they are also known as blood blisterlike aneurysms (BBLA) ${ }^{2}$ or ICA anterior wall aneurysms ${ }^{3}$. They are rare, comprising 0.9 to $6.5 \%$ of all ICA aneurysms ${ }^{3}$.

These lesions are fairly small, sometimes appearing as just a protrusion of the vessel wall (expressed as being "blood blister-like"). They grow rapidly in a short time and easily rupture, especially during surgery. Eventually they cannot be eliminated easily by the ordinary clipping procedure requiring wrapping or encircling methods ${ }^{4-6}$.

Curiously these aneurysms are more often reported by oriental authors ${ }^{1-5,7-9}$. We will present a 37-year male Brazilian case successfully treated with clip application.

\section{CASE}

A 37-year-old man presented with sudden onset of severe headache, vomiting and briefly consciousness disturbance. Examination revealed mild meningismus, but no focal neurological deficits. A CT scan of the brain revealed thick and diffuse subarachnoid hemorrhage $(\mathrm{SAH})$ involving the carotid cistern and left sylvian fissure. The angiogram revealed a small irregularity of the ventral left supraclinoid artery suspicious for a BBLA.(Fig 1)

We performed a left pterional craniotomy, with micro dissection and exposure of the optic nerve and the ICA. The lateral fissure was widely opened allowing visualization of ICA bifurcation, middle and anterior cerebral arteries. There were signs of hemorrhage in the carotid cistern and sylvian fissure. The clot was carefully dissected away from the optic nerve and ICA in order to approach the involved segment. The aneurysm resembled a focal dissection, with no emerging vessels in neighborhood. Parallel application of a titanium straight angle clip was performed without temporary clipping of ICA. Care was taken to extend the clip beyond the aneurysm margins with minimal ICA stenosis (Figs 2 and 3).

The post-operative period was uneventful and the patient was discharged after control angiogram which revealed completely exclusion of the aneurysm and ICA patency (Fig 4).

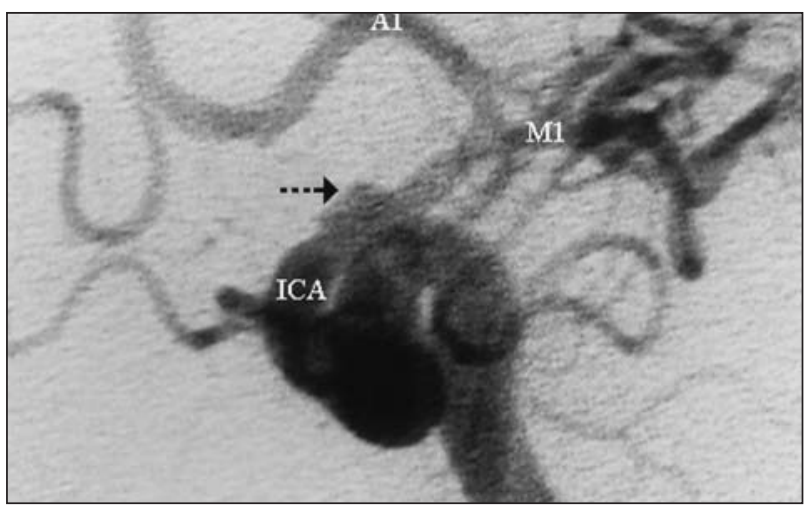

Fig 1. Left carotid angiogram. Note small aneurysm (arrow) on the dorsal surface of ICA. ICA, left internal carotid artery; A7, left anterior cerebral artery; $\mathrm{M1}$, left middle cerebral artery.

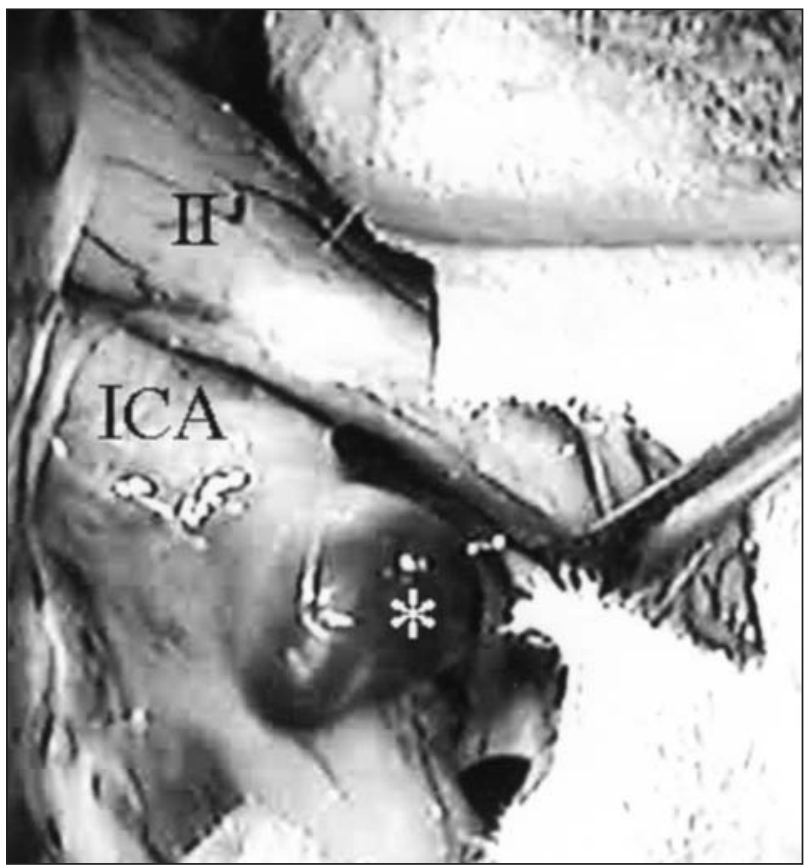

Fig 2. Microphotograph. Microdissection showing the blood blisterlike aneurysm located at a nonbranching region of ICA. Note the close relationship with optical nerve. II, left optical nerve; ICA, left internal carotid artery; *aneurysm.

\footnotetext{
ANEURISMA DA CARÓTIDA INTERNA NÃO RELACIONADA A RAMO PERFURANTE

Restauração Hospital, Recife PE, Brazil: 'Neurosurgeon, ${ }^{1}$ Neurosurgery Resident.

Received 13 February 2008, received in final form 17 Abril 2008. Accepted 17 May 2008.

Dr. Joacil Carlos da Silva - Rua Agenor Lopes 424/701 - 51021-110 Recife PE - Brasil. E-mail: joacil_carlos@hotmail.com
} 


\section{DISCUSSION}

BBLA have some unique characteristics that make distinction from the common saccular or berry aneurysms $\mathrm{s}^{1-4,6-8}$.

Rhoton introduced three rules related to the anatomy of saccular aneurysms that should be considered when planning the operative approach to these lesions. First, these aneurysms arise at a branching site on the parent artery. Second, saccular aneurysms arise at a turn or curve in the artery. Saccular aneurysms arise on the convex, not concave, side of the curve. Third, saccular aneurysms point in the direction that the blood would have gone if the curve at the aneurysm site were not present ${ }^{10}$.

BBLA contradict the first Rhoton's rule as they are frequently located at the dorsal or anterior wall of ICA, a nonbranching region ${ }^{3}$.

The histological characteristics of BBLA include focal wall defects covered with clot and fibrous tissue. The focal wall defects may be the result of laceration of the ICA wall caused by ulceration and penetration into the internal elastica lamina, resulting from arteriosclerosis. The walls of BBLA are composed of only normal adventitia, in an abrupt transformation from the sclerotic ICA wall. In contrast, ordinary berry aneurysms are formed by thickened intima and/or adventitia, with rich collagen and inflammatory cells, that is continuous with the three-layered arterial wall ${ }^{2,3}$.

Therefore, arteriosclerosis alone may not explain the causative mechanism for BBLA. Associated hypertension, ulceration resulting from arteriosclerosis, arterial dissection and hemodynamic stress may also influence the occurrence of these aneurysms ${ }^{1,3}$.

Dissection of the ICA was often observed in patients with BBLA but not in patients with saccular-type aneurysms, suggesting the presence of specific pathological features in BBLA. Hemodynamic stress is thought to initiate berry aneurysms and may also influence the occurrence of $B B L A^{1-4}$.

The treatment options include direct lesion clipping ${ }^{6}$, wrapping or encircling techniques ${ }^{4,8}$ and endovascular obliteration ${ }^{9}$ of the ICA with or without bypass procedures. ${ }^{3}$

The main surgical problem is the extremely fragile nature of the walls and the usually large defect in the artery. The direct clip application could lead to avulsion of the aneurysm or to obliteration of the parent vessel. Reconstruction techniques may involve direct suture ${ }^{5}$, Sundt clip application $^{6}$, vascular titanium clamps and bypass procedures ${ }^{3}$.

Parallel clipping of the ICA (including the normal arterial wall beyond the lesion), under decreased pressure produced by temporary clamping of the cervical ICA, is recommended, to avoid breakage of the transitional zone

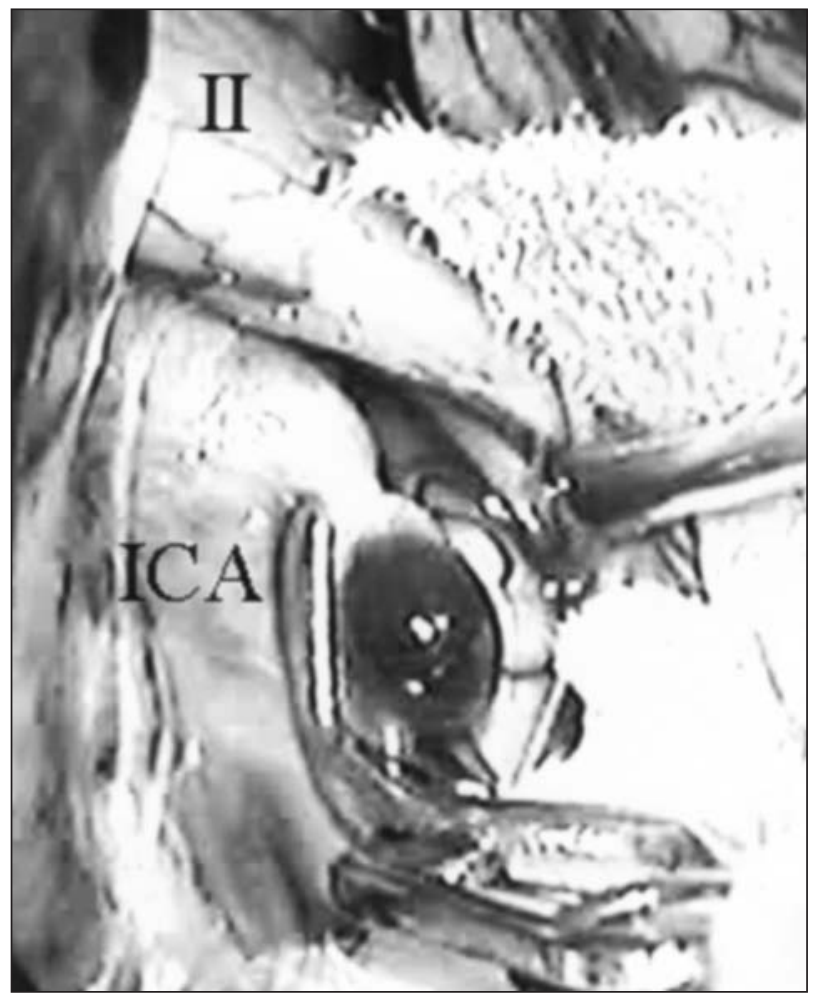

Fig 3. Microphotograph. Parallel clipping of the ICA was possible extending the blades of the clip beyond arterial wall defect. II, left optical nerve; ICA, left internal carotid artery.

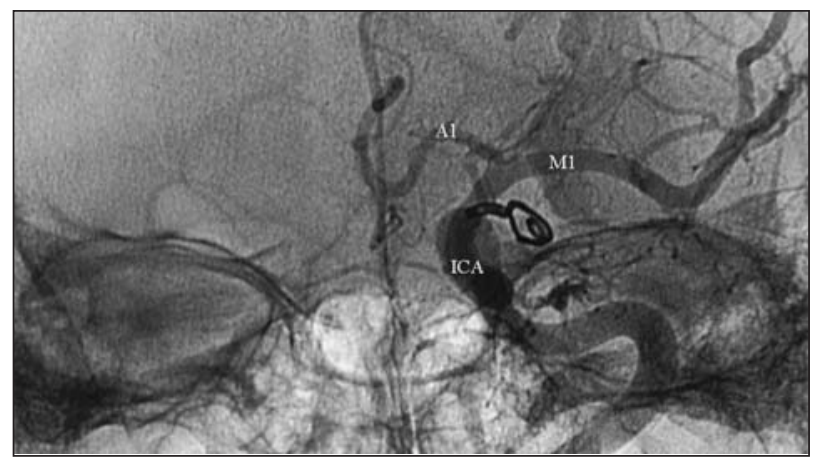

Fig 4. Post operative carotid angiogram. Note absence of ICA stenosis. ICA, left internal carotid artery; Al, left anterior cerebral artery; $\mathrm{M1}$, left middle cerebral artery.

between the normal ICA and the aneurysm wall ${ }^{3}$. Another clip option is the Sundt encircling clip developed for direct reconstruction of $\mathrm{ICA}^{6}$.

The classic wrapping method with cotton or muscle and fibrin glue does not prevent rebleeding. The modern encircling techniques combine synthetic wrapping materials secured with titanium clips around the artery and apparently are effective in reinforce the transitional zone. Complications of rebleeding/regrowth and the risk of perforator injury, as well as difficulty in identifying the border of the wrapping, are problems associated with this method ${ }^{4,5,8}$. 
While avoiding these dangerous lesions, proximal ICA occlusion by endovascular or surgical means may be a treatment option, but this does not eliminate rebleeding risk posed by retrograde flow $w^{1,3}$.

Technically, the endovascular occlusion of focal ICA segments is not easy ${ }^{9}$. Unlike aneurysm clips, the precise and stable placement of endovascular devices such as coils and balloons is difficult. For compact and precise trapping, balloons may be preferred to coils, but ICA BBLA are very fragile and usually do not have uniform configurations that accommodate balloons. In a small series of six patients endovascular treated, there was regrowth in all cases ${ }^{7,9}$.

In conclusion, the blood blister-like aneurysms are a rare but important cause of subarachnoid hemorrhage. The early recognition of these lesions is crucial to adequate treatment planning. Neurosurgeons should attempt to the fragility and extension of arterial wall defect as well to the rather elevated risks of regrowth and rebleeding.

Nowadays, microsurgery with arterial wall reconstruction (direct clip or encircling techniques) is preferable to endovascular treatment.

\section{REFERENCES}

1. Nakagawa F, Kobayashi S, Takemae T, Sugita K. Aneurysms protruding from the dorsal wall of the internal carotid artery. J Neurosurg 1986; 65:303-308.

2. Ishikawa T, Nakamura N, Houkin K, Nomura M. Pathological consideration of a "blister-like" aneurysm at the superior wall of the internal carotid artery: case report. Neurosurgery 1997;40:403-406.

3. Ogawa A, Suzuki M, Ogasawara K. Aneurysms at nonbranching sites in the supraclinoid portion of the internal carotid artery: internal carotid artery trunk aneurysms. Neurosurgery 2000;47:578-586.

4. Kubo Y, Ogasawara K, Tomitsuka N, Otawara Y, Watanabe M, Ogawa A. Wrap-clipping with polytetrafluoroethylene for ruptured blisterlike aneurysms of the internal carotid artery. J Neurosurg 2006;105:785-787.

5. Joo S, Kim T, Moon K, et al. Arterial suturing followed by clip reinforcement with circumferential wrapping for blister-like aneurysms of the internal carotid artery. Surg Neurol 2006;66:424-429.

6. Sekula R, Cohen D, Quigley M, Jannetta P. Primary treatment of a blister-like aneurysm with an encircling clip graft: technical case report. Neurosurgery 2006; 59[ONS Suppl 1]:ONS-168.

7. Tanoue S, Kyosue H, Matsumoto S, Yamashita M, Nagatomi H, Mori H. Ruptured "blisterlike" aneurysm with a pseudoaneurysm formation requiring delayed intervention with endovascular coil embolization. J Neurosurg 2004;101:159-162.

8. Yanaka K, Meguro K, Nose T. Repair of a tear at the base of a blisterlike aneurysm with suturing and an encircling clip: technical note. Neurosurgery 2002;50:218-221.

9. Park J, Park I, Han D, et al. Endovascular treatment of blood blister-like aneurysms of the internal carotid artery. J Neurosurg 2007;106:812-819.

10. Rhoton Jr. A. Aneurysms. Neurosurgery 2002;51(Suppl 1):S121-S158. 